\title{
Genome-wide RNAi analysis reveals that simultaneous inhibition of specific mevalonate pathway genes potentiates tumor cell death
}

\author{
Aleksandra A. Pandyra 1,2,4, Peter J. Mullen ${ }^{1}$, Carolyn A. Goard ${ }^{1,2}$, Elke Ericson ${ }^{3,5}$, \\ Piyush Sharma ${ }^{4}$, Manpreet Kalkat ${ }^{1,2}$, Rosemary Yu ${ }^{1,2}$, Janice T. Pong ${ }^{1,2}$, \\ Kevin R. Brown ${ }^{3}$, Traver Hart ${ }^{3}$, Marinella Gebbia ${ }^{3}$, Karl S. Lang ${ }^{4}$, Guri Giaever ${ }^{3,6}$, \\ Corey Nislow ${ }^{3,6}$, Jason Moffat ${ }^{3}$, Linda Z. Penn ${ }^{1,2}$ \\ ${ }^{1}$ Princess Margaret Cancer Centre, Toronto, ON, Canada \\ ${ }^{2}$ Department of Medical Biophysics, University of Toronto, Toronto, ON, Canada \\ ${ }^{3}$ Donnelly Centre and Banting \& Best Department of Medical Research, University of Toronto, Toronto, Canada \\ ${ }^{4}$ Institute of Immunology, Medical Faculty, University of Duisburg-Essen, Essen, Germany \\ ${ }^{5}$ Now located at AstraZeneca R\&D, Mölndal Sweden \\ ${ }^{6}$ Now located at Faculty of Pharmaceutical Sciences, University of British Columbia, Vancouver, BC, Canada \\ Correspondence to: \\ Linda Z. Penn, e-mail: Ipenn@uhnres.utoronto.ca \\ Keywords: SREBP2, statins, mevalonate pathway, feedback inhibition, tumor metabolism \\ Received: June 17, $2015 \quad$ Accepted: August 12, $2015 \quad$ Published: August 22, 2015
}

\section{ABSTRACT}

The mevalonate (MVA) pathway is often dysregulated or overexpressed in many cancers suggesting tumor dependency on this classic metabolic pathway. Statins, which target the rate-limiting enzyme of this pathway, 3-hydroxy-3-methylglutaryl-CoA reductase (HMGCR), are promising agents currently being evaluated in clinical trials for anti-cancer efficacy. To uncover novel targets that potentiate statin-induced apoptosis when knocked down, we carried out a pooled genome-wide short hairpin RNA (shRNA) screen. Genes of the MVA pathway were amongst the top-scoring targets, including sterol regulatory element binding transcription factor 2 (SREBP2), 3-hydroxy-3-methylglutaryl-coenzyme A synthase 1 (HMGCS1) and geranylgeranyl diphosphate synthase 1 (GGPS1). Each gene was independently validated and shown to significantly sensitize A549 cells to statin-induced apoptosis when knocked down. SREBP2 knockdown in lung and breast cancer cells completely abrogated the fluvastatin-induced upregulation of sterol-responsive genes HMGCR and HMGCS1. Knockdown of SREBP2 alone did not affect three-dimensional growth of lung and breast cancer cells, yet in combination with fluvastatin cell growth was disrupted. Taken together, these results show that directly targeting multiple levels of the MVA pathway, including blocking the sterol-feedback loop initiated by statin treatment, is an effective and targetable anti-tumor strategy.

\section{INTRODUCTION}

Cancer cells undergo drastic metabolic reprogramming to meet their increased demand for energy and macromolecules. Amongst the metabolic changes occurring in cancer cells is increased de novo lipid and cholesterol synthesis through both the fatty acid synthesis and mevalonate (MVA) pathways [1,2]. The latter not only leads to the production of cholesterol, but also results in important non-sterol end products including farnesyl and geranylgeranyl isoprenoids, dolichol, ubiquinone, and isopentenyladenine (Figure 1A).

In normal cells, the MVA pathway is highly regulated, however, this pathway can be dysregulated in tumor cells by a variety of mechanisms. Tumors frequently have altered metabolism of glucose, glutamine or acetate, 


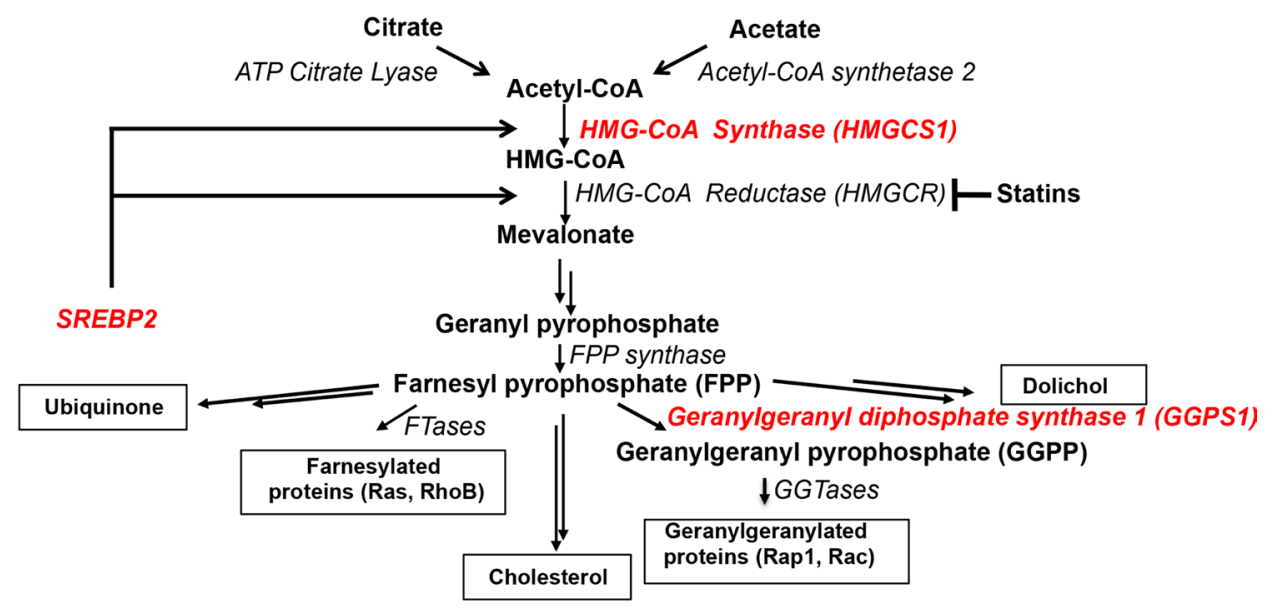

B
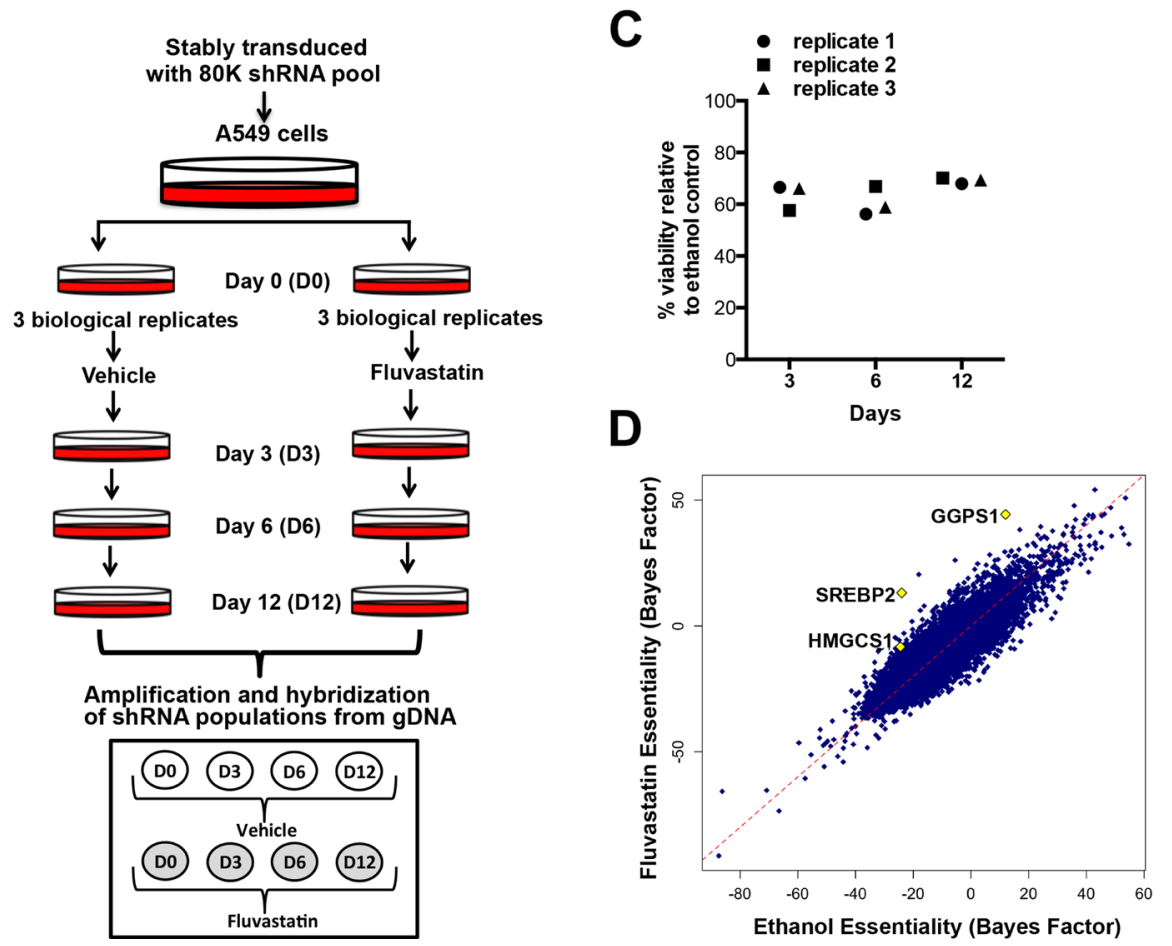

Figure 1: A genome-wide dropout screen uncovers putative shRNAs that potentiate fluvastatin-induced cell death. A. A simplified schematic of the mevalonate (MVA) pathway. Double arrows represent multiple steps in the pathway. B. In the pooled, genome-wide dropout screen A549 cells that had been stably transduced with the library of $80 \mathrm{~K}$ shRNAs were treated with sublethal doses of fluvastatin (4-5 $\mu \mathrm{M})$ every 3 days, over 12 days. C. Viability as assessed by trypan blue exclusion, relative to control of the fluvastatin-treated replicates. D. Significant hits, HMGCS1, GGPS1 and SREBP2, chosen for follow-up are indicated on the scatterplot (diamonds).

which can lead to increased acetyl-CoA, the substrate of the MVA pathway. Solid tumors also often have upregulated ATP citrate lyase and acetyl-CoA synthase 2, both of which produce acetyl-CoA [1-5]. In addition, MVA pathway enzymes can be upregulated by mutant p53 [6] and their elevated expression is associated with poor prognosis and reduced survival in cancer patients [6,7]. Consistent with this observation, over-expression of the rate-limiting enzyme, 3-hydroxy-3-methylglutaryl-CoA reductase (HMGCR), contributes to oncogenic progression [7]. Furthermore, the restorative feedback response typically found in normal cells is deficient in some tumor cells [8-11]. These multiple levels of MVA pathway dysregulation suggest that cancer cells are particularly dependent on the MVA-derived end products and therefore preferentially sensitive to inhibition of the MVA pathway. 
Statins inhibit the MVA pathway and have been successfully used for decades in the control of hypercholesterolemia. Understanding the production and homeostatic regulation of the MVA pathway in normal cells has been instrumental in the development of these effective, well-tolerated cholesterol control agents. Statins inhibit HMGCR leading to the depletion of intracellular cholesterol $[12,13]$. This triggers a restorative feedback response mediated by the sterol regulatory element binding protein 2 (SREBP2), which induces the transcription of genes such as HMGCR and low-density lipoprotein receptor (LDLr) $[14,15]$. In the liver, this leads to cellular uptake of LDL and the depletion of serum cholesterol levels.

Accumulating epidemiological evidence [16-18] and prospective clinical trials in cancer [19-22] indicate that statins have potential as anti-cancer agents. Evidence suggests that statins can also trigger tumor cells to undergo apoptosis [20, 23-25]. As approved agents, statins can be fast-tracked to impact cancer patient care and targeting the MVA pathway is therefore an important and emerging therapeutic strategy. Cancer therapeutics are not typically used as single agents, but rather delivered as drug cocktails to increase inhibitory activity. To identify novel sensitizers that could combine to maximize the anti-cancer efficacy of statins, we performed a pooled, genome-wide shRNA dropout screen. The A549 cancer cell line was stably transduced with the RNAi Consortium (TRC1) shRNA library [26-28] and exposed to vehicle control or sublethal doses of fluvastatin. Genes required for cell survival in the fluvastatin-treated cells were identified using bioinformatics methods as previously described [29]. The top scoring hits included the MVA pathway related genes geranylgeranyl diphosphate synthase 1 (GGPS1), 3-hydroxy-3-methylglutaryl-CoA synthase 1 (HMGCS1), and SREBP2.

Subsequent validation demonstrated that individual knockdown of GGPS1, HMGCS1 or SREBP2 in combination with fluvastatin treatment had anti-proliferative and pro-apoptotic activity. Further characterization revealed that fluvastatin-sensitive lung and breast cancer cells stably expressing shRNAs targeting SREBP2 lost the ability to upregulate HMGCR and HMGCS1 in response to fluvastatin treatment. Furthermore, three-dimensional (3D) growth of these cells knocked down for SREBP2 expression was disrupted following statin exposure, indicating that simultaneously targeting HMGCR and SREBP2 is a promising novel anti-tumor therapeutic strategy. In conclusion, we identified vulnerabilities of the MVA pathway and potential new therapeutic targets that, in combination with statins, can be exploited in the treatment of lung and breast cancer. This paradigm of simultaneous targeting multiple genes within a metabolic pathway is likely instructive for effective targeting of other metabolic tumor vulnerabilities.

\section{RESULTS}

To identify novel sensitizers that potentiate statininduced anti-proliferative activity and maximize anticancer efficacy, we designed a pooled genome-wide dropout shRNA screen (Figure 1B). Lung carcinoma A549 cells were used in this screen as they are relatively insensitive to statin-induced apoptosis. Cells stably expressing shRNA were treated with sublethal (defined as $\sim 30-40 \%$ reduction in cell viability, Figure $1 \mathrm{C}$ ) doses of fluvastatin. Cells sensitive to the combined chemical and genetic perturbation dropped out from the population over the 12 days of the experiment (Figure 1B and 1C). Analysis of the shRNAs remaining in the ethanol controltreated but not fluvastatin-treated cells identified genes that were lethal in cells exposed to fluvastatin. Because A549 cells are relatively statin-insensitive, uncovering statin sensitizers in these cells has the potential to identify vulnerabilities that may be targeted to increase statin anti-cancer activity in tumors otherwise unresponsive to statins. Of 283 significantly under-represented shRNAs following fluvastatin treatment (Supplementary Table 1), a number of genes among the top hits included several related to the MVA pathway (highlighted in red), including HMGCS1, GGPS1 and SREBP2 (Figure 1A and 1D).

HMGCS1 is upstream of HMGCR and synthesizes HMG-CoA, the substrate used by HMGCR in the production of MVA [30]. GGPS1 lies downstream of HMGCR and catalyzes the synthesis of geranylgeranyl pyrophosphate (GGPP), which is used for the post-translational prenylation of many proteins, including small GTP-binding proteins such as the Rho family of proteins integral for cell survival, growth and cytoskeletal organization [31, 32]. SREBP2 plays a critical role in regulating the MVA pathway. In response to intracellular cholesterol depletion, SREBP2 is cleaved and translocates to the nucleus, where it induces the transcription of sterol-responsive genes such as HMGCR and HMGCS1 [14, 15, 33].

We validated potential hits from our shRNA screen by generating A549 cell lines knocked down for HMGCS1, GGPS1 and SREBP2, using two unique shRNA's for each target. These cell lines are referred to as shHMGCS1 A, shHMGCS1 B, shGGPS1 A, shGGPS1 B, shSREBP2 A and shSREBP2 B, respectively. Control cell lines expressing shRNAs targeting non-human genes (e.g. LacZ) were similarly generated and assayed (termed shControl). mRNA levels were robustly reduced by approximately 80 percent in the shSREBP2, shGGPS1 and shHMGCS1 cell lines relative to shControl lines (Figure 2A) as were the protein levels in the shSREBP2 and shHMGCS1 cell lines (Figure 2B). GGPS1 protein levels were not assessed due to the lack of specific antibodies.

The shSREBP2 and shGGPS1 cell lines were particularly sensitive to the anti-cancer effects of fluvastatin and this was reflected in the reduction of IC50 values relative to shControl lines (Figure 2C). 


\section{A}

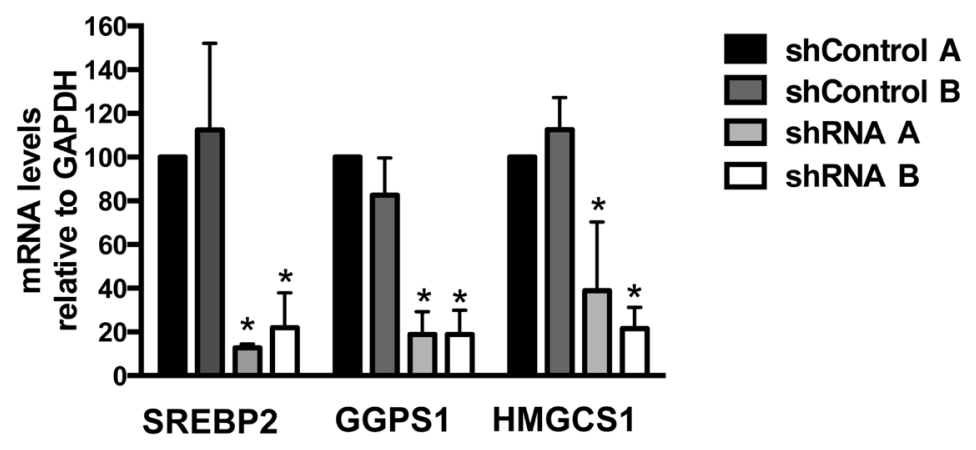

B

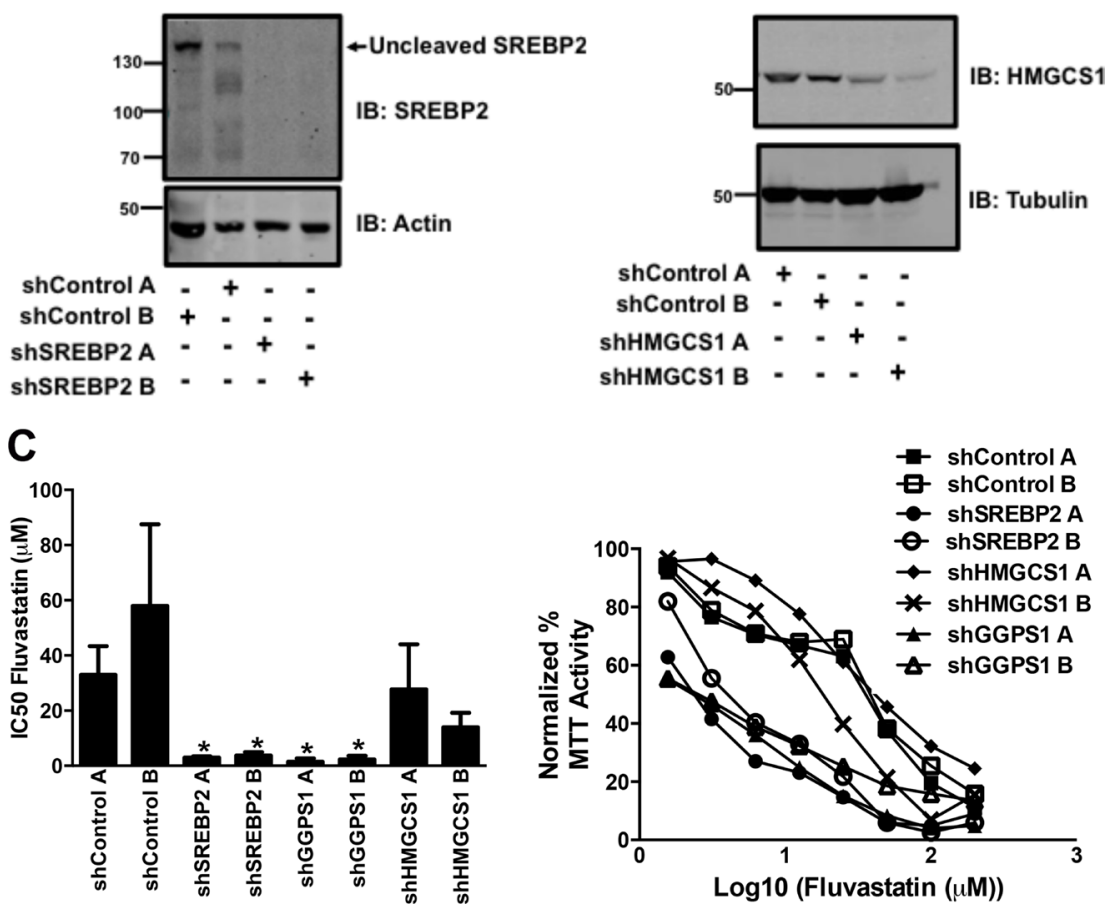

Figure 2: Generation of A549 cell lines stably expressing shRNA constructs targeting genes of the MVA pathway. A-B. RNA and protein lysates were harvested from asynchronously growing A549 cells that express two independent control shRNAs (shControl A, shControl B) or shRNAs targeting SREBP2, GGPS1 or HMGCS1 (shRNA A, shRNA B). Real-time mRNA EXPRESSION data represents the mean $\pm \mathrm{SD}$ of three independent experiments. Immunoblots are representative of at least three independent experiments. C. Knockdown of SREBP2 and GGPS1 significantly decreased fluvastatin IC50 values. ${ }^{*} p<0.05$ (one-way ANOVA with Tukey post-test). Representative dose-response curves assessed using the MTT assay are shown in C. (right panel).

When shSREBP2, shGGPS1 and shHMGCS1 A549 cell lines were treated with a sub-lethal dose of fluvastatin, we observed a dose-dependent induction of apoptosis as measured by cellular pre-G1 DNA content (Figure 3A). Apoptosis in the shControl fluvastatin-treated cells was minimal. The initial genome-wide results, combined with their independent validation confirms these gene products as targets whose knockdown in combination with fluvastatin administration increases tumor cell apoptosis.

The stable knockdown of SREBP2, GGPS1 and HMGCS1 can potentially perturb other pathways in addition to the MVA pathway. To verify that the apoptosis was MVA pathway dependent, we co-administered either MVA or GGPP with fluvastatin across our panel of shRNA cell lines. Fluvastatin-induced apoptosis was reversed by the co-administration of MVA or GGPP in the shSREBP2 and shHMGCS1 cell lines (Figure 3B) indicating that the apoptosis was dependent on MVA pathway inhibition and specifically dependent on the GGPP-producing arm of the MVA pathway. This result independently validates the functional importance of protein geranylgeranylation in the regulation of tumor cell death, and is consistent with previous reports showing that statin-induced apoptosis of several tumor types can be functionally blocked by the exogenous addition of MVA or GGPP $[8,25,34]$. Co-administration of GGPP (but not 


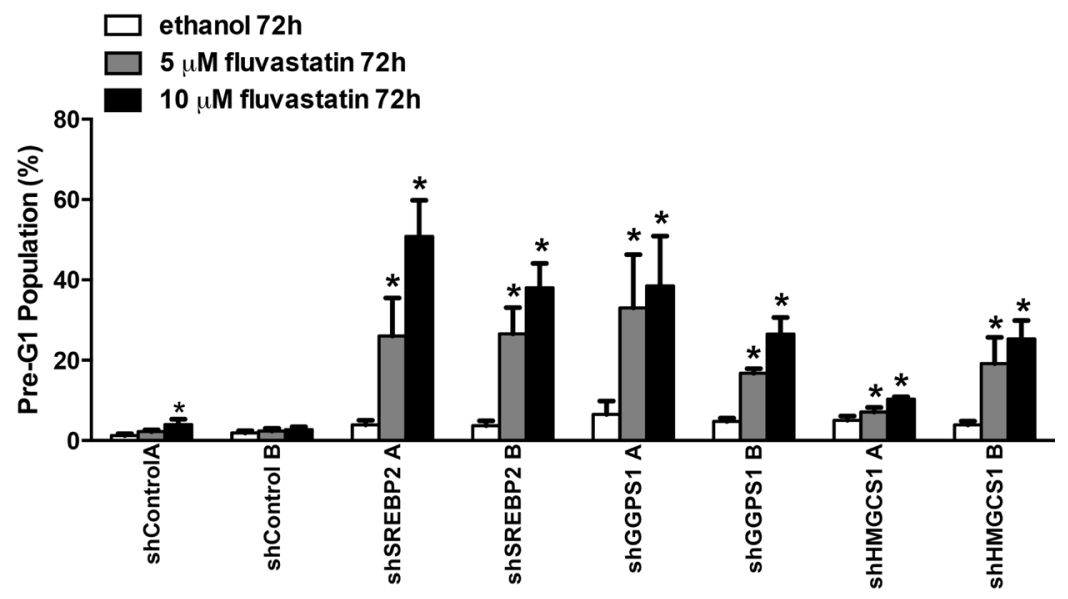

B

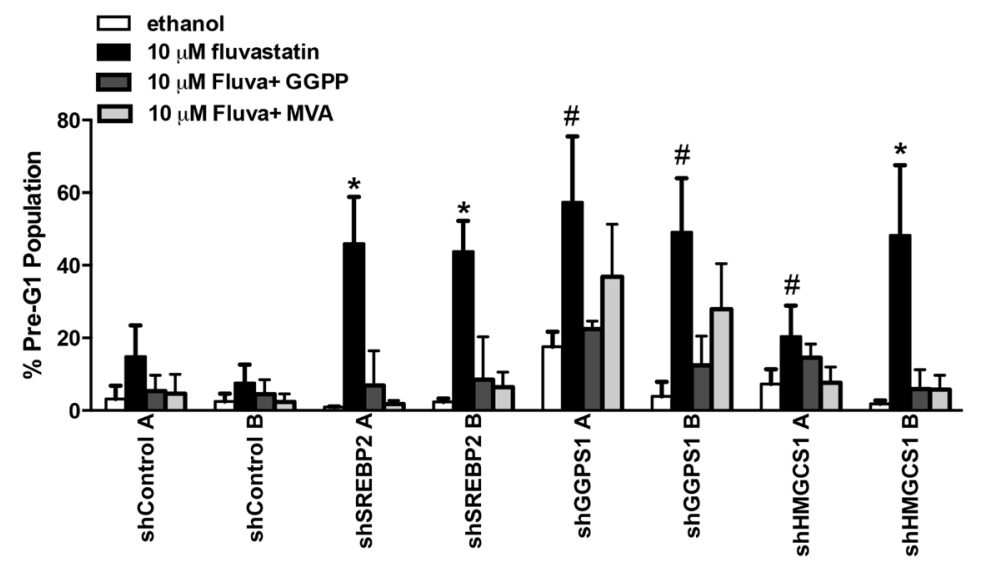

C

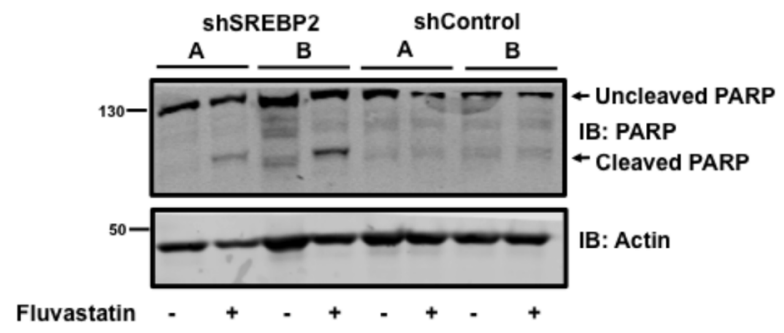

Figure 3: shRNAs targeting MVA pathway gene hits potentiate fluvastatin-induced apoptosis. A. Exposure to fluvastatin $(10 \mu \mathrm{M})$ for 72 hours of A549 cell lines stably expressing the indicated shRNA significantly induced apoptosis. B. Fluvastatin-induced apoptosis 72 hours post-treatment in the shGGPS1 cell lines was reversed by the concomitant addition of GGPP (10 $\mu \mathrm{M})$ and not MVA $(100 \mu \mathrm{M})$ but by both GGPP and MVA in shSREBP2 and shHMGCS1 cell lines. Bars represent the mean \pm SD of at least three independent experiments * \# $p<0.05$ (*one-way ANOVA with Tukey-post test within each shA549 cell line comparing all groups, the fluvastatintreated group being significantly different from the others, \# the fluvastatin-treated group being significantly different than the ethanol and fluvastatin + GGPP groups). C. Protein lysates harvested from A549 cell lines treated with $10 \mu \mathrm{M}$ of fluvastatin for 72 hours were immunoblotted and probed for PARP expression. Immunoblots are representative of at least three independent experiments.

MVA) to fluvastatin-treated shGGPS1 cell lines blocked fluvastatin-induced apoptosis (Figure 3B). The absence of effect seen with co-administered MVA was expected because MVA production occurs several steps upstream of the GGPS1-catalyzed GGPP production. Providing additional evidence of apoptosis, treatment of shSREBP2 cell lines but not shControl A549 cells with fluvastatin induced PARP cleavage, which is an indicator of apoptosis downstream of caspase activation (Figure 3C). Taken together, knockdown of specific MVA pathway genes (SREBP2, GGPS1 and HMGCS1) can potentiate statininduced apoptosis in A549 cells.

To evaluate the nature and generality of our results, we chose to further focus on the combination of statins with the knockdown of SREBP2, which not only decreased fluvastatin IC50 values but also triggered robust 
apoptosis. Furthermore, SREBP2 plays a central role in the MVA pathway as a master transcriptional activator of sterol responsive genes including HMGCS1 and HMGCR $[12,15]$. To this end, we first tested whether SREBP2 knockdown also sensitized cells of another solid tumor type to fluvastatin-induced apoptosis. We chose breast cancer, as recent window-of-opportunity, pre-operative clinical trials showed that lipophilic statins (fluvastatin or atorvastatin) can impact tumor growth, suggesting statins may have a role in the management of this disease [19, 20]. The triple negative basal-like MDA-MB-231 and luminal MCF7 cells are relatively sensitive and insensitive to statin-induced apoptosis, respectively [35]. As previously observed with the A549 cells, MDA-MB-231 and MCF7 breast cancer cell lines stably expressing shRNAs targeting SREBP2 displayed increased fluvastatin sensitivity expressed as anti-proliferative and pro-apoptotic effects
(Figure 4A-4F). SREBP2 knockdown alone in all cell lines tested did not perturb the rate of cell growth (assessed by doubling times) (Figure 5A). Furthermore, the sensitization effect was fluvastatin-specific as SREBP2 knockdown did not sensitize lung and breast cancer cells to the anti-cancer effects of doxorubicin, an anti-cancer drug with a distinct mechanism of action compared to fluvastatin (Figure 5B). Taken together, knockdown of SREBP2 specifically potentiated the anti-cancer effects of fluvastatin in lung and breast cancer cells.

The sensitizing effects mediated by SREBP2 knockdown were completely reversed by co-administration of either MVA or GGPP. This functional rescue supports the model that SREBP2-mediated effects on the MVA pathway were responsible for the observed phenotype. Upon HMGCR inhibition and subsequent cholesterol depletion, SREBP2 initiates the transcription
A

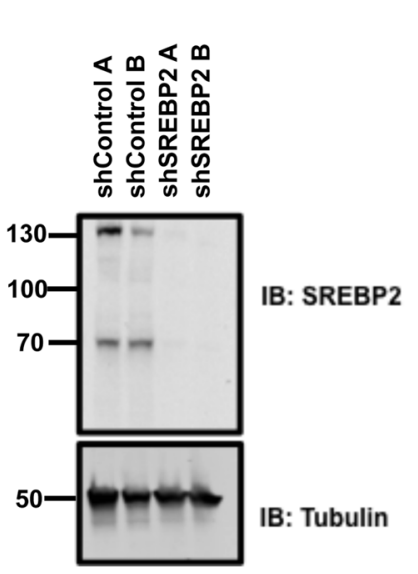

D

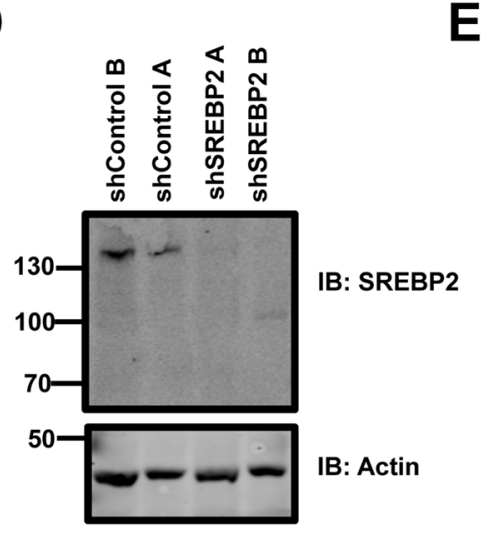

E
B
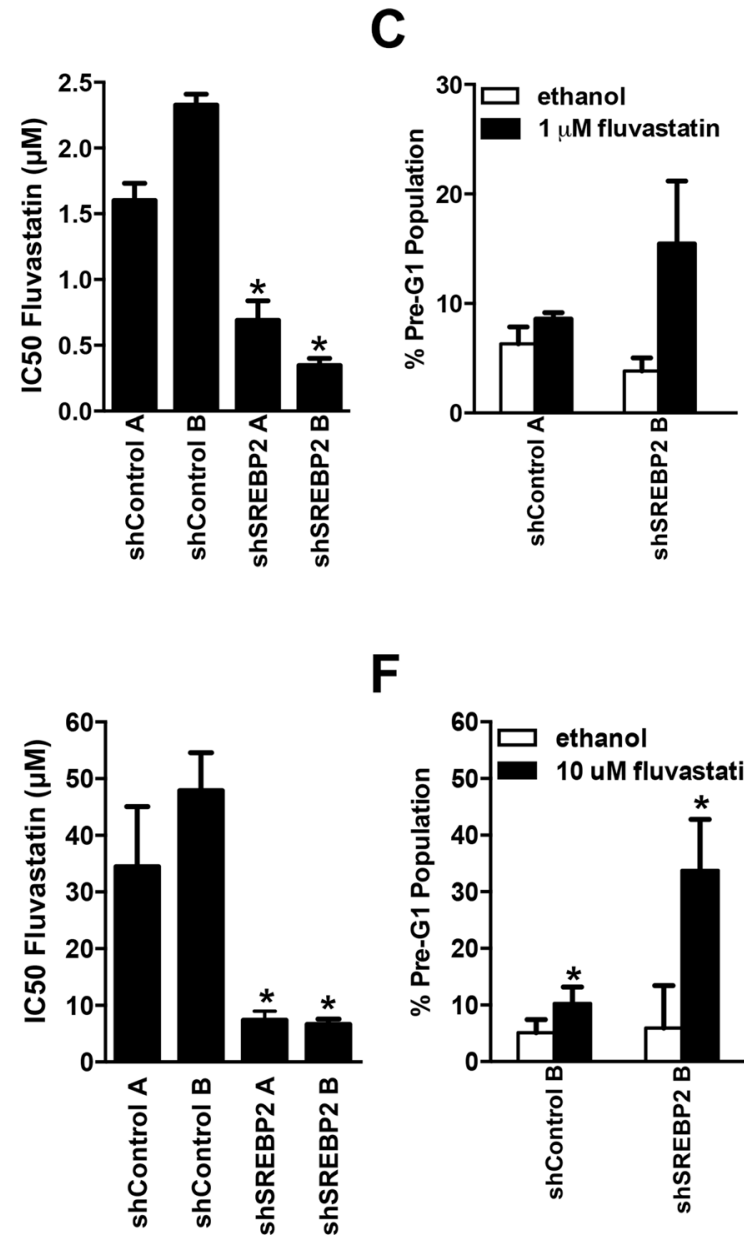

$\mathbf{F}$

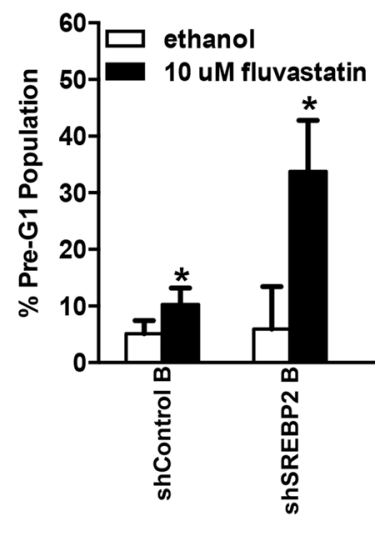

Figure 4: Stable knockdown of SREBP2 sensitizes breast cancer MDA-MB-231 and MCF7 cells to the pro-apoptotic and anti-proliferative effects of fluvastatin. A-F. Protein lysates harvested from asynchronously growing MDA-MB-231 and MCF7 cells were immunoblotted for SREBP2 protein expression relative to cells transduced with the shControls (A and D respectively). Fluvastatin IC50 values in the MDA-MB-231 and MCF7 cell lines expressing the SREBP2 shRNAs were significantly lower when compared to the control cell lines (B and E respectively). Exposure of representative MDA-MB-231 and MCF7 cell lines to sub-lethal doses of fluvastatin caused a significant increase in apoptosis ( $\mathrm{C}$ and $\mathrm{F}$ respectively). ${ }^{*} p<0.05$ ( $t$-test, unpaired, two-tailed, comparison made within each cell line). Immunoblots represent a minimum of two independent experiments. 

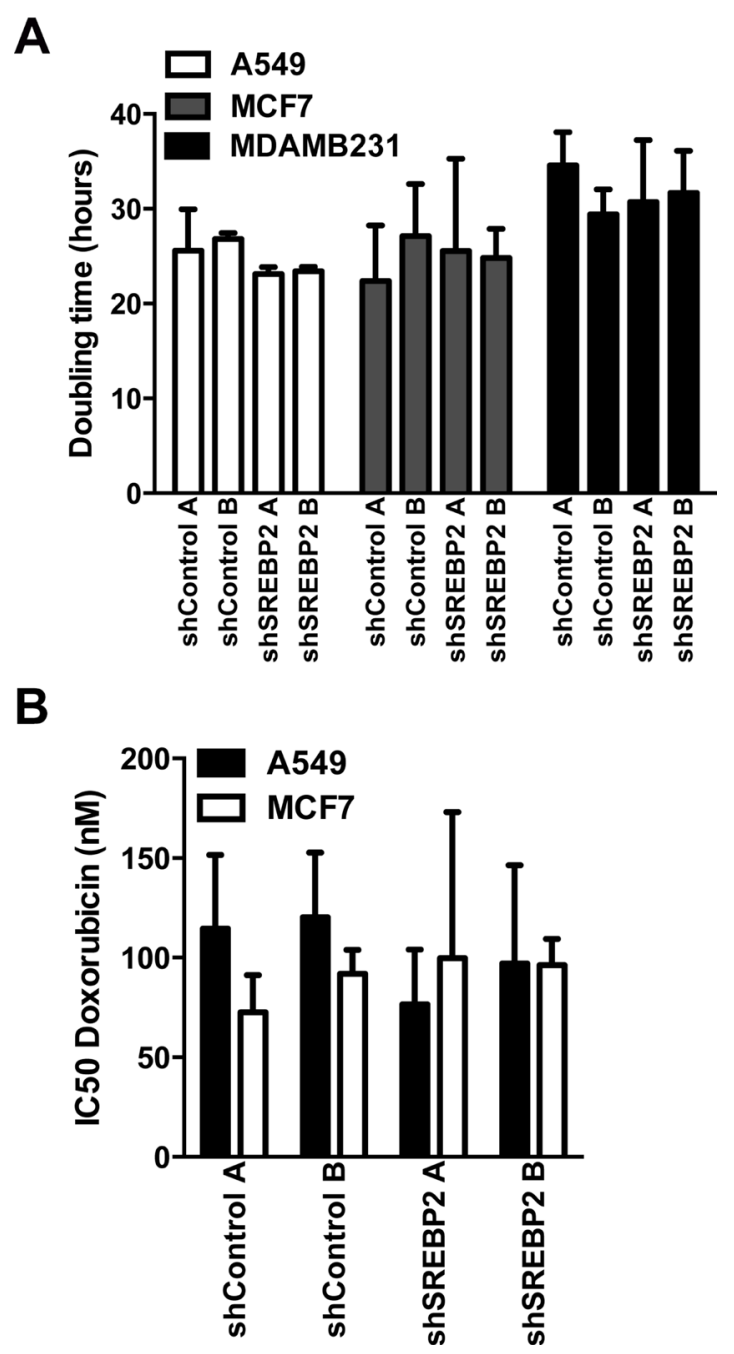

Figure 5: Knockdown of MVA pathway genes does not sensitize breast and lung cancer cells to the anti-proliferative effects of doxorubicin. A. Proliferation as assessed by measuring the doubling time using the CyQuant cell proliferation assay kit was not significantly different between cell lines expressing the SREBP2 shRNAs and control cell lines in A549, MCF7 and MDA-MB-231 cells (one-way ANOVA with Tukey post test within each shA549 cell line comparing all groups). Bars represent the mean \pm SD of at least three independent experiments. B. Doxorubicin IC50 values of the A549 and MCF7 cell lines expressing the SREBP2 shRNAs were not significantly different when compared to the cell lines expressing control shRNAs.

of sterol-responsive genes. This has been observed in many cancer cells and has been postulated to be an impediment to statin therapy $[23,36]$. Upon fluvastatin treatment, HMGCR and HMGCS1 were significantly upregulated in the shControl A549 cells (Figure 6A and $6 \mathrm{~B}$ ). This was also observed in the MCF7 breast cancer cells (Figure 7A). The upregulation of HMGCR and HMGCS1 was accompanied by increased SREBP2 cleavage in the shControl lines (Figure 6C). The sterolfeedback response initiated upon HMGCR inhibition and mediated by SREBP2 cleavage was sufficient to blunt the anti-proliferative effects of fluvastatin but, as expected, was successfully abrogated with the knockdown of SREBP2 in shSREBP2 cell lines. In 3D culture growth, fluvastatin treatment disrupted the morphology and structure of the shSREBP2 A549 cells (Figure 6D) and shSREBP2 MCF7 cells [35, 37] (Figure 7B).

\section{DISCUSSION}

By conducting a pooled, genome-wide shRNA screen, we have demonstrated the utility of targeting more than one member of the MVA pathway to further sensitize cells to fluvastatin. HMGCS1, GGPS1 and SREBP2 knockdown, in concert with statin-mediated HMGCR inhibition, can robustly enhance tumor cell apoptosis.

Targeting HMGCS1 in combination with fluvastatin is attractive in tumor cells where HMGCS1 is robustly upregulated in response to statin treatment as was the case in the lung and breast cancer cell lines used in our 

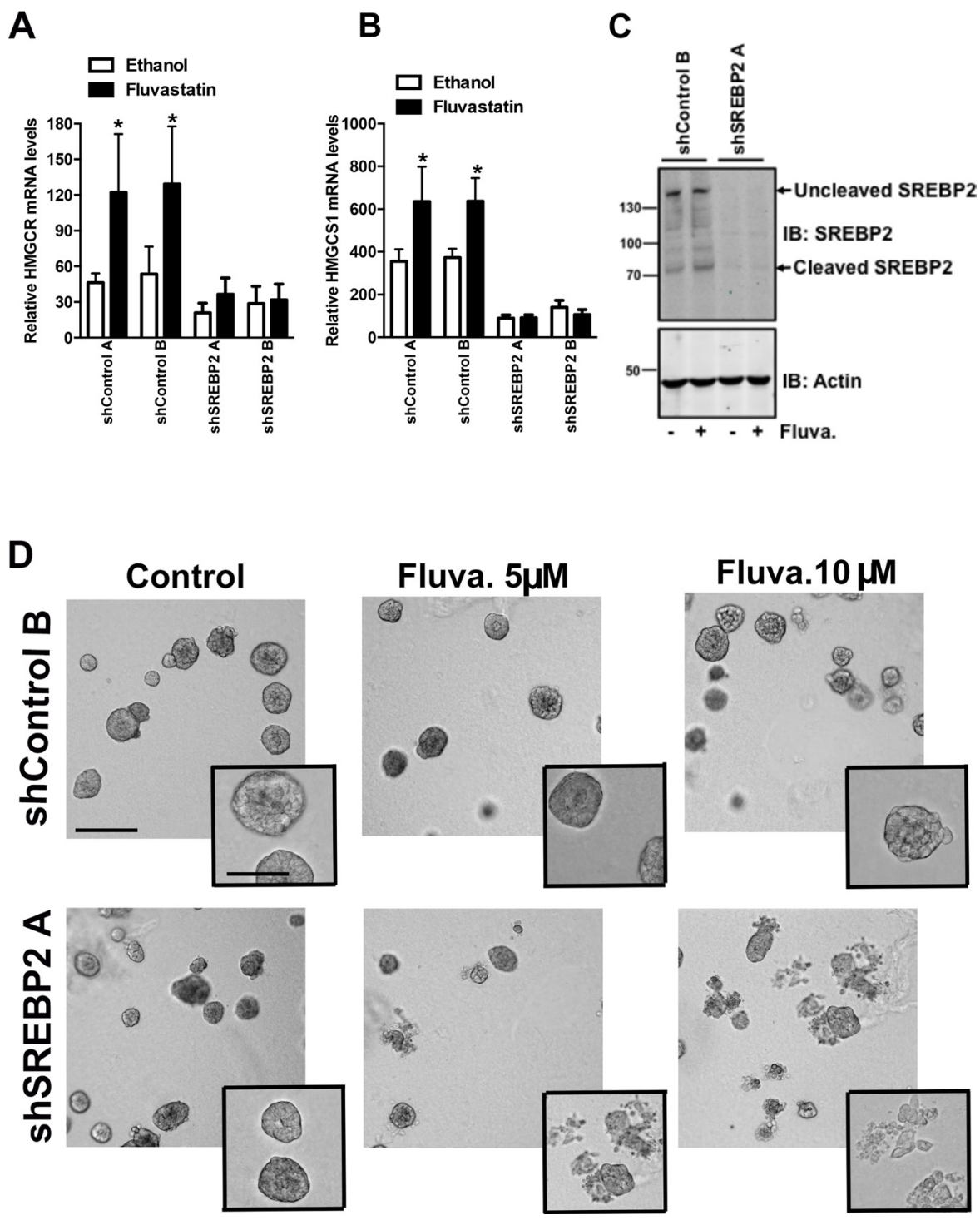

Figure 6: Knockdown of SREBP2 abrogates the sterol-feedback loop and impairs 3D growth upon fluvastatin treatment. Cells were treated for 24 hours with $10 \mu \mathrm{M}$ of fluvastatin or ethanol control and assessed for expression of A. HMGCR B. HMGCS1 relative to GAPDH in shA549 cell lines. Bars represent the mean $\pm \mathrm{SD}$ of at least three independent experiments $* p<0.05$ ( $t$-test, unpaired, two-tailed, comparison made within each cell line). C. Protein lysates harvested from A549 cell lines treated with $10 \mu \mathrm{M}$ of fluvastatin (Fluva.) for 24 hours were immunoblotted for SREBP2 expression. Immunoblots are representative of at least three independent experiments. D. Treatment with fluvastatin for 72 hours with the indicated fluvastatin concentrations disrupts A549 acini in shSREBP2 cell lines in 3D culture. Cells were imaged using an AxioObserver and are representative of three independent experiments. Scale bar represents $200 \mu \mathrm{m}$ in main image and $100 \mu \mathrm{m}$ in inset.

study. In addition, analysis of cancer genomics datasets using cBioPortal $[38,39]$ shows that HMGCS1 can be amplified in cancers, including breast and lung (Figure 8). This suggests tumors with elevated basal HMGCS1 expression may be particularly responsive to treatment with fluvastatin and an inhibitor of HMGCS1.

Our finding that knocking down GGPS1 potentiates fluvastatin- mediated HMGCR inhibition is consistent with previous results from us and others that this arm of the MVA pathway is functionally critical for statin-induced apoptosis of tumor cells [25] Moreover, recent results demonstrate dramatic synergy of pharmacological GGPS1 inhibitors was evident when combined with statins at inducing leukemia cell kill [40, 41]. GGPS1 is an emerging novel therapeutic target that provides an alternative to the existing, but not highly efficacious single agent farnesyl and gernaylgeranyltransferase (GGTase) inhibitors designed to target isoprenoid biosynthesis [42, 43]. This inspires a novel model by which the statintriggered feedback loop would restore the MVA endproducts, but in the presence of GGPS1 inhibitors, this lethal vulnerability would not be rescued leading to tumor 
A
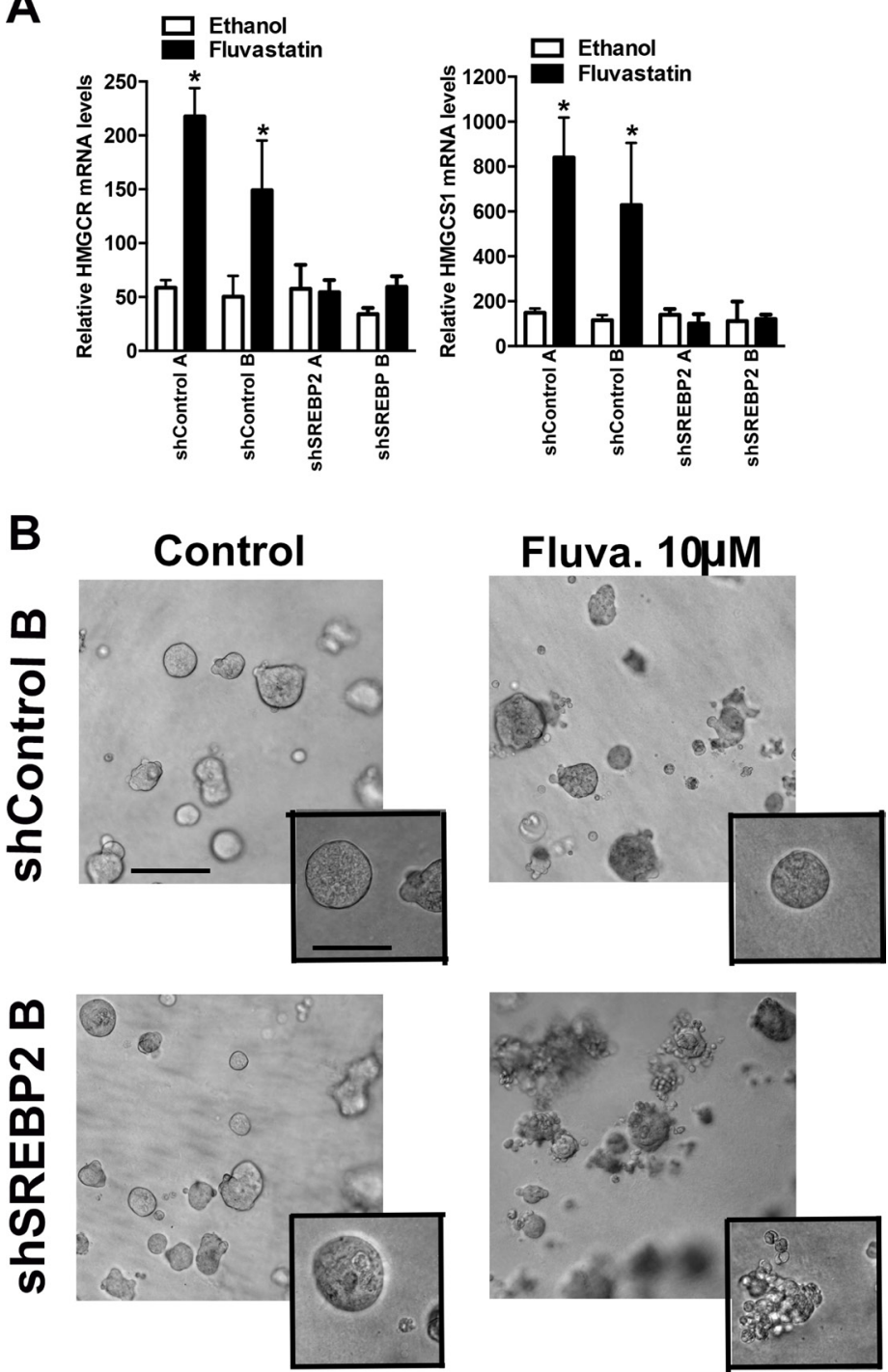

Figure 7: Knockdown of SREBP2 abrogates the sterol-feedback loop and impairs 3D growth of MCF7 cells upon fluvastatin treatment. A. Cells were treated for 24 hours with $10 \mu \mathrm{M}$ of fluvastatin or ethanol control and assessed for expression of HMGCR (A), HMGCS1 (B) relative to GAPDH in shMCF7 cell lines. Bars represent the mean \pm SD of at least three independent experiments $* p<0.05$ ( $t$-test, unpaired, two-tailed, comparison made within each cell line). B. Treatment with fluvastatin for 72 hours with the indicated concentrations disrupts MCF7 acini in the shSREBP2 cell line in 3D culture but not in the shControl cell line. Images are representative of three independent experiments composed of three technical replicates. Scale bar represents $200 \mu \mathrm{m}$ in main image and $100 \mu \mathrm{m}$ in inset.

cell death. As HMGCR is the rate-limiting enzyme of the MVA pathway, its inhibition should lead to a depletion of all products downstream of MVA. Unexpectedly, we observed a strong fluvastatin potentiation upon knockdown of specific genes in the pathway (GGPS1 and HMGCS1) and further study is required to delineate the balance between HMGCR inhibition and the counteracting effects of the sterol-feedback loop.

In normal cells, the de novo lipid synthesis pathways are under tight physiological control by the sterol response element binding protein (SREBP) family of transcription factors, SREBP1 and SREBP2. Although the physiological role of SREBP2 in maintaining cholesterol homeostasis is well defined, the importance of this basic helix-loophelix leucine zipper transcription factor in cancer cells and oncogenesis remains relatively unexplored unlike that of SREBP1, which is highly activated in various cancers and its activation is linked with the PI3K/Akt/mTOR signalling axis [44-46]. SREBP2 is over-expressed during progression in primary prostate cancer cells [46], and combined SREBP2 and SREBP1 knockdown in cancer cell lines caused endoplasmic reticulum stress and induction of apoptosis in lipoprotein-depleted conditions [47]. We found that SREBP2 knockdown did not affect cell growth in lung and breast cancer cells, and that differences between the shSREBP2 and shControl cell lines were only apparent 


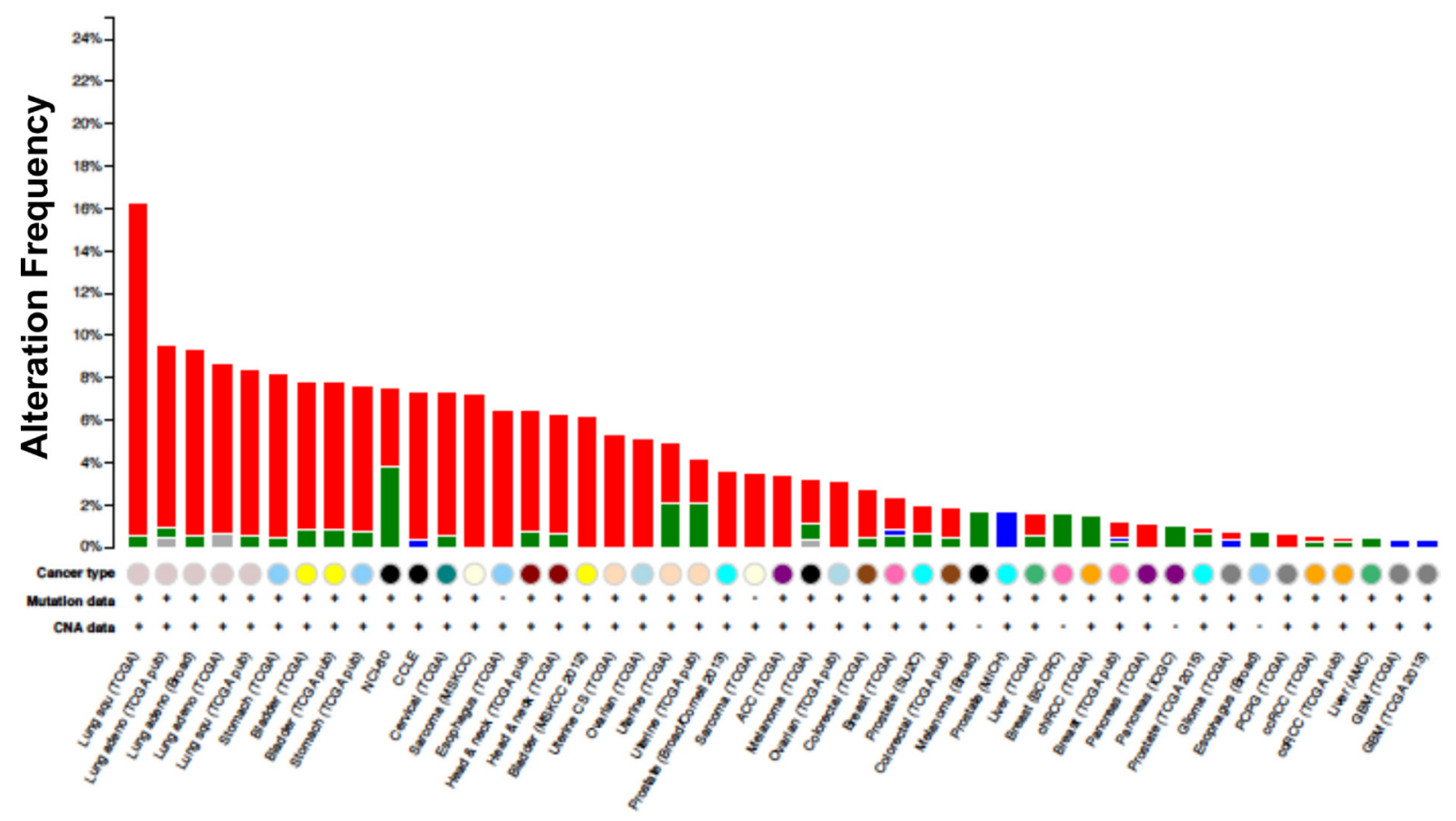

Mutation Deletion Amplification Multiple Alterations

Figure 8: HMGCS1 is amplified in various cancers. Analysis of cancer genomics datasets using cBioPortal demonstrating HMGCS1 genomic alterations across various tumor types.

following fluvastatin exposure. Although no specific SREBP2 inhibitors are available, we recently identified another drug, dipyridamole, that effectively blocked SREBP2 cleavage and synergized with statins to induce apoptosis in haematological malignancies indicating that SREBP2 is a novel promising therapeutic target [36].

Targeting SREBP2 effectively suppresses the restorative feedback loop triggered by statin treatment, widening the therapeutic window to include tumors otherwise unresponsive to statins. Therefore, SREBP2 is an especially attractive and novel target to have emerged from the screen. Statins are safe, FDA-approved and off-patent, making them cost-effective anti-cancer agents that may be explored in future combination chemotherapies, and here, we have identified three novel targets augmenting their efficacy. In addition, given the inherent homeostatic feedback loops that are built into the metabolic circuitry of a cell, the concept of blocking feedback loops that blunt the efficacy of initial pathway inhibition are important as more anti-cancer agents targeting abnormal tumor metabolism are developed.

\section{MATERIALS AND METHODS}

\section{Cell culture}

Cell lines were maintained in Dulbecco's Modified Eagle Medium was supplemented with $10 \%$ fetal bovine serum (GIBCO) and penicillin-streptomycin.

\section{shRNA screen, lentivirus and shRNA cell line generation}

A549 cell line, stably transduced with the RNAi Consortium (TRC1) shRNA library [27] was treated with fluvastatin (US Biologicals) over 12 days. Genomic DNA (gDNA) was amplified and shRNA populations hybridized onto custom Affymetrix Gene Modulation Array Platform (GMAP) arrays. Differences between shRNA abundances over 12 days were assessed using the Bayes Factor [29]. For generation of stably expressing shRNA cell lines, DNA from bacterial glycerol stocks of shRNAs from the TRC1 library was amplified using E.coli and purified (Qiagen Plasmid Maxiprep kit). Lentiviral particles were generated by calcium phosphate transfection of sub-confluent (50-60\%) HEK293TV cells with $10 \mu \mathrm{g}$ of TRC pLKO.1 puro shRNA construct, $5 \mu \mathrm{g}$ each of pMDG1.vsvg, pRSV-Rev and $\mathrm{pMDLg} / \mathrm{pRRE}$ constructs. Lentiviral particles were collected 24 and 48 hours later, filtered though a $0.45 \mu \mathrm{m}$ filter and stored at $-80^{\circ} \mathrm{C}$. Parental cell lines were infected with lentiviral particles containing the indicated shRNAs and puromycin selected ( $48 \mathrm{hrs}, 2 \mu \mathrm{g} / \mathrm{ml}$ of puromycin).

\section{Immunoblotting}

For SREBP2, HMGCS1 and PARP protein detection, cells were lysed using boiling SDS lysis buffer (1.1\% SDS, $11 \%$ glycerol, $0.1 \mathrm{M}$ Tris $\mathrm{pH} 6.8)$ with $10 \% \beta$-mercaptoethanol). Blots were probed with 
anti-SREBP2 (BD Pharmingen), anti-HMGCS1 (Santa Cruz Biotechnology), anti-PARP (Cell Signaling) antitubulin (Santa Cruz Biotechnology) and anti-actin (Sigma) and detected using the Odyssey infrared imaging system (LI-COR Biosciences).

\section{MTT, fixed propidium iodide (PI) and CyQuant proliferation assays}

MTT (Sigma-Aldrich) assays were done as previously described [23, 36]. Briefly, 1.5-2.0 $\times$ $10^{4}$ cells $/ \mathrm{ml}$ were plated in 96-well plates and after 24 hours, treated with a range of fluvastatin and doxorubicin concentrations for 72 hours. Half-maximal inhibitory concentrations (IC50) values were computed from dose-response curves using Prism (v5.0, GraphPad Software). For fixed PI assays, cells were fixed in $70 \%$ ethanol, treated with DNAse-free RNAse and stained with PI (Sigma-Aldrich). Cells were analyzed for the dying pre-G1 population by flow cytometry. Cell proliferation assays were carried out as per manufacturer's protocol (Life Technologies).

\section{Real-time PCR}

RNA was harvested from asynchronously growing cells and isolated using Trizol (Invitrogen). mRNA knockdown of SREBP2 (Hs01081784_m1), GGPS1 (Hs00191442_m1) and HMGCS1 (HS00266810_m1) relative to GAPDH (Hs99999905_m1) were assessed using TaqMan Gene Expression Assays using TaqMan master mix (Applied Biosystems). HMGCR mRNA levels were assayed as previously described [23].

\section{D Morphogenesis}

Performed as previously described [35].

\section{ACKNOWLEDGMENTS}

We would like to thank all members of the Penn lab for helpful review of the manuscript. This work was undertaken in part, thanks to funding from the CRC Program (L.Z.P), the OICR through funding provided by the Province of Ontario (L.Z.P.), the CBCF (A.P., C.A.G, M.K.), the Knudson Fellowship (P.J.M.), and the Ontario Ministry of Health and Long Term Care. The views expressed do not necessarily reflect those of the Ontario Ministry of Health and Long Term Care.

\section{CONFLICTS OF INTEREST}

The authors declare no conflict of interest.

\section{REFERENCES}

1. Mashima T, Seimiya H, Tsuruo T. De novo fatty-acid synthesis and related pathways as molecular targets for cancer therapy. British journal of cancer. 2009; 100:1369-1372.

2. Zaidi N, Swinnen JV, Smans K. ATP-citrate lyase: a key player in cancer metabolism. Cancer research. 2012; 72:3709-3714.

3. Comerford SA, Huang Z, Du X, Wang Y, Cai L, Witkiewicz AK, Walters $\mathrm{H}$, Tantawy $\mathrm{MN}, \mathrm{Fu} \mathrm{A}$, Manning HC, Horton JD, Hammer RE, McKnight SL, Tu BP. Acetate dependence of tumors. Cell. 2014; 159:1591-1602.

4. Mashimo T, Pichumani K, Vemireddy V, Hatanpaa KJ, Singh DK, Sirasanagandla S, Nannepaga S, Piccirillo SG, Kovacs Z, Foong C, Huang Z, Barnett S, Mickey BE, DeBerardinis RJ, Tu BP, Maher EA, et al. Acetate is a bioenergetic substrate for human glioblastoma and brain metastases. Cell. 2014; 159:1603-1614.

5. Schug ZT, Peck B, Jones DT, Zhang Q, Grosskurth S, Alam IS, Goodwin LM, Smethurst E, Mason S, Blyth K, McGarry L, James D, Shanks E, Kalna G, Saunders RE, Jiang M, et al. Acetyl-CoA synthetase 2 promotes acetate utilization and maintains cancer cell growth under metabolic stress. Cancer cell. 2015; 27:57-71.

6. Freed-Pastor WA, Mizuno $\mathrm{H}$, Zhao X, Langerod A, Moon SH, Rodriguez-Barrueco R, Barsotti A, Chicas A, Li W, Polotskaia A, Bissell MJ, Osborne TF, Tian B, Lowe SW, Silva JM, Borresen-Dale AL, et al. Mutant p53 disrupts mammary tissue architecture via the mevalonate pathway. Cell. 2012; 148:244-258.

7. Clendening JW, Pandyra A, Boutros PC, El Ghamrasni S, Khosravi F, Trentin GA, Martirosyan A, Hakem A, Hakem R, Jurisica I, Penn LZ. Dysregulation of the mevalonate pathway promotes transformation. Proceedings of the National Academy of Sciences of the United States of America. 2010; 107:15051-15056.

8. Wong WW, Clendening JW, Martirosyan A, Boutros PC, Bros C, Khosravi F, Jurisica I, Stewart AK, Bergsagel PL, Penn LZ. Determinants of sensitivity to lovastatin-induced apoptosis in multiple myeloma. Mol Cancer Ther. 2007; 6:1886-1897.

9. Tatidis L, Gruber A, Vitols S. Decreased feedback regulation of low density lipoprotein receptor activity by sterols in leukemic cells from patients with acute myelogenous leukemia. Journal of lipid research. 1997; 38:2436-2445.

10. Vitols S, Gahrton G, Bjorkholm M, Peterson C. Hypocholesterolaemia in malignancy due to elevated low-density-lipoprotein-receptor activity in tumour cells: evidence from studies in patients with leukaemia. Lancet. $1985 ; 2: 1150-1154$

11. Chen Y, Hughes-Fulford M. Human prostate cancer cells lack feedback regulation of low-density lipoprotein receptor and its regulator, SREBP2. International journal of cancer Journal international du cancer. 2001; 91:41-45. 
12. Brown MS, Goldstein JL. Multivalent feedback regulation of HMG CoA reductase, a control mechanism coordinating isoprenoid synthesis and cell growth. J Lipid Res. 1980; 21:505-517.

13. Kita T, Brown MS, Goldstein JL. Feedback regulation of 3-hydroxy-3-methylglutaryl coenzyme A reductase in livers of mice treated with mevinolin, a competitive inhibitor of the reductase. The Journal of clinical investigation. 1980; 66:1094-1100.

14. Brown MS, Goldstein JL. The SREBP pathway: regulation of cholesterol metabolism by proteolysis of a membranebound transcription factor. Cell. 1997; 89:331-340.

15. Brown MS, Goldstein JL. Cholesterol feedback: from Schoenheimer's bottle to Scap's MELADL. J Lipid Res. 2009; 50 Suppl:S15-27.

16. Ahern TP, Pedersen L, Tarp M, Cronin-Fenton DP, Garne JP, Silliman RA, Sorensen HT, Lash TL. Statin prescriptions and breast cancer recurrence risk: a Danish nationwide prospective cohort study. Journal of the National Cancer Institute. 2011; 103:1461-1468.

17. Khurana V, Bejjanki HR, Caldito G, Owens MW. Statins reduce the risk of lung cancer in humans: a large case-control study of US veterans. Chest. 2007; 131:1282-1288.

18. Farwell WR, Scranton RE, Lawler EV, Lew RA, Brophy MT, Fiore LD, Gaziano JM. The association between statins and cancer incidence in a veterans population. Journal of the National Cancer Institute. 2008; 100:134-139.

19. Bjarnadottir O, Romero Q, Bendahl PO, Jirstrom K, Ryden L, Loman N, Uhlen M, Johannesson H, Rose C, Grabau D, Borgquist S. Targeting HMG-CoA reductase with statins in a window-of-opportunity breast cancer trial. Breast cancer research and treatment. 2013; 138:499-508.

20. Garwood ER, Kumar AS, Baehner FL, Moore DH, Au A, Hylton N, Flowers CI, Garber J, Lesnikoski BA, Hwang ES, Olopade O, Port ER, Campbell M, Esserman LJ. Fluvastatin reduces proliferation and increases apoptosis in women with high grade breast cancer. Breast cancer research and treatment. 2010; 119:137-144.

21. Advani AS, McDonough S, Copelan E, Willman C, Mulford DA, List AF, Sekeres MA, Othus M, Appelbaum FR. SWOG0919: a Phase 2 study of idarubicin and cytarabine in combination with pravastatin for relapsed acute myeloid leukaemia. British journal of haematology. 2014.

22. Hus M, Grzasko N, Szostek M, Pluta A, Helbig G, Woszczyk D, Adamczyk-Cioch M, Jawniak D, Legiec W, Morawska M, Kozinska J, Wacinski P, Dmoszynska A. Thalidomide, dexamethasone and lovastatin with autologous stem cell transplantation as a salvage immunomodulatory therapy in patients with relapsed and refractory multiple myeloma. Annals of hematology. 2011; 90:1161-1166.

23. Clendening JW, Pandyra A, Li Z, Boutros PC, Martirosyan A, Lehner R, Jurisica I, Trudel S, Penn LZ. Exploiting the mevalonate pathway to distinguish statin-sensitive multiple myeloma. Blood. 2010; 115:4787-4797.

24. Wong WW, Dimitroulakos J, Minden MD, Penn LZ. HMG$\mathrm{CoA}$ reductase inhibitors and the malignant cell: the statin family of drugs as triggers of tumor-specific apoptosis. Leukemia. 2002; 16:508-519.

25. Xia Z, Tan MM, Wong WW, Dimitroulakos J, Minden MD, Penn LZ. Blocking protein geranylgeranylation is essential for lovastatin-induced apoptosis of human acute myeloid leukemia cells. Leukemia. 2001; 15:1398-1407.

26. Moffat J, Grueneberg DA, Yang X, Kim SY, Kloepfer AM, Hinkle G, Piqani B, Eisenhaure TM, Luo B, Grenier JK, Carpenter AE, Foo SY, Stewart SA, Stockwell BR, Hacohen N, Hahn WC, et al. A lentiviral RNAi library for human and mouse genes applied to an arrayed viral highcontent screen. Cell. 2006; 124:1283-1298.

27. Blakely K, Ketela T, Moffat J. Pooled lentiviral shRNA screening for functional genomics in mammalian cells. Methods in molecular biology. 2011; 781:161-182.

28. Marcotte R, Brown KR, Suarez F, Sayad A, Karamboulas K, Krzyzanowski PM, Sircoulomb F, Medrano M, Fedyshyn Y, Koh JL, van Dyk D, Fedyshyn B, Luhova M, Brito GC, Vizeacoumar FJ, Vizeacoumar FS, et al. Essential gene profiles in breast, pancreatic, and ovarian cancer cells. Cancer discovery. 2012; 2:172-189.

29. Hart T, Brown KR, Sircoulomb F, Rottapel R, Moffat J. Measuring error rates in genomic perturbation screens: gold standards for human functional genomics. Molecular systems biology. 2014; 10:733.

30. Mathews ES, Mawdsley DJ, Walker M, Hines JH, Pozzoli M, Appel B. Mutation of 3-hydroxy-3-methylglutaryl CoA synthase I reveals requirements for isoprenoid and cholesterol synthesis in oligodendrocyte migration arrest, axon wrapping, and myelin gene expression. The Journal of neuroscience: the official journal of the Society for Neuroscience. 2014; 34:3402-3412.

31. Ericsson J, Greene JM, Carter KC, Shell BK, Duan DR, Florence C, Edwards PA. Human geranylgeranyl diphosphate synthase: isolation of the cDNA, chromosomal mapping and tissue expression. Journal of lipid research. 1998; 39:1731-1739.

32. Park J, Matralis AN, Berghuis AM, Tsantrizos YS. Human isoprenoid synthase enzymes as therapeutic targets. Frontiers in chemistry. 2014; 2:50.

33. Hua X, Yokoyama C, Wu J, Briggs MR, Brown MS, Goldstein JL, Wang X. SREBP-2, a second basic-helixloop-helix-leucine zipper protein that stimulates transcription by binding to a sterol regulatory element. Proceedings of the National Academy of Sciences of the United States of America. 1993; 90:11603-11607.

34. Yanae $M$, Tsubaki $M$, Satou $T$, Itoh $T$, Imano $M$, Yamazoe Y, Nishida S. Statin-induced apoptosis via the suppression of ERK1/2 and Akt activation by inhibition of the geranylgeranyl-pyrophosphate biosynthesis in 
glioblastoma. Journal of experimental \& clinical cancer research: CR. 2011; 30:74.

35. Goard CA, Chan-Seng-Yue M, Mullen PJ, Quiroga AD, Wasylishen AR, Clendening JW, Sendorek DH, Haider S, Lehner R, Boutros PC, Penn LZ. Identifying molecular features that distinguish fluvastatin-sensitive breast tumor cells. Breast cancer research and treatment. 2014; 143:301-312.

36. Pandyra A, Mullen PJ, Kalkat M, Yu R, Pong JT, Li Z, Trudel S, Lang KS, Minden MD, Schimmer AD, Penn LZ. Immediate utility of two approved agents to target both the metabolic mevalonate pathway and its restorative feedback loop. Cancer research. 2014; 74:4772-4782.

37. Kenny PA, Lee GY, Myers CA, Neve RM, Semeiks JR, Spellman PT, Lorenz K, Lee EH, Barcellos-Hoff MH, Petersen OW, Gray JW, Bissell MJ. The morphologies of breast cancer cell lines in three-dimensional assays correlate with their profiles of gene expression. Molecular oncology. 2007; 1:84-96.

38. Cerami E, Gao J, Dogrusoz U, Gross BE, Sumer SO, Aksoy BA, Jacobsen A, Byrne CJ, Heuer ML, Larsson E, Antipin Y, Reva B, Goldberg AP, Sander C, Schultz N. The cBio cancer genomics portal: an open platform for exploring multidimensional cancer genomics data. Cancer discovery. 2012; 2:401-404.

39. Gao J, Aksoy BA, Dogrusoz U, Dresdner G, Gross B, Sumer SO, Sun Y, Jacobsen A, Sinha R, Larsson E, Cerami E, Sander C, Schultz N. Integrative analysis of complex cancer genomics and clinical profiles using the cBioPortal. Science signaling. 2013; 6:pl1.

40. Dudakovic A, Wiemer AJ, Lamb KM, Vonnahme LA, Dietz SE, Hohl RJ. Inhibition of geranylgeranyl diphosphate synthase induces apoptosis through multiple mechanisms and displays synergy with inhibition of other isoprenoid biosynthetic enzymes. The Journal of pharmacology and experimental therapeutics. 2008; 324:1028-1036.
41. Wiemer AJ, Wiemer DF, Hohl RJ. Geranylgeranyl diphosphate synthase: an emerging therapeutic target. Clinical pharmacology and therapeutics. 2011; 90:804-812.

42. Tsimberidou AM, Chandhasin C, Kurzrock R. Farnesyltransferase inhibitors: where are we now? Expert opinion on investigational drugs. 2010; 19:1569-1580.

43. Martin NE, Brunner TB, Kiel KD, DeLaney TF, Regine WF, Mohiuddin M, Rosato EF, Haller DG, Stevenson JP, Smith D, Pramanik B, Tepper J, Tanaka WK, Morrison B, Deutsch P, Gupta AK, et al. A phase I trial of the dual farnesyltransferase and geranylgeranyltransferase inhibitor L-778, 123 and radiotherapy for locally advanced pancreatic cancer. Clinical cancer research : an official journal of the American Association for Cancer Research. 2004; 10:5447-5454.

44. Porstmann T, Santos CR, Griffiths B, Cully M, Wu M, Leevers S, Griffiths JR, Chung YL, Schulze A. SREBP activity is regulated by $\mathrm{mTORC} 1$ and contributes to Aktdependent cell growth. Cell metabolism. 2008; 8:224-236.

45. Guo D, Reinitz F, Youssef M, Hong C, Nathanson D, Akhavan D, Kuga D, Amzajerdi AN, Soto H, Zhu S, Babic I, Tanaka K, Dang J, Iwanami A, Gini B, Dejesus J, et al. An LXR agonist promotes glioblastoma cell death through inhibition of an EGFR/AKT/SREBP-1/LDLRdependent pathway. Cancer discovery. 2011; 1:442-456.

46. Ettinger SL, Sobel R, Whitmore TG, Akbari M, Bradley DR, Gleave ME, Nelson CC. Dysregulation of sterol response element-binding proteins and downstream effectors in prostate cancer during progression to androgen independence. Cancer research. 2004; 64:2212-2221.

47. Griffiths B, Lewis CA, Bensaad K, Ros S, Zhang Q, Ferber EC, Konisti S, Peck B, Miess H, East P, Wakelam M, Harris AL, Schulze A. Sterol regulatory element binding protein-dependent regulation of lipid synthesis supports cell survival and tumor growth. Cancer \& metabolism. 2013; 1:3. 